Supporting Information

\title{
Self-bonded Hydrogel Inverse Opal Particles as Sprayed Flexible Patch for Wound Healing
}

Li Wang ${ }^{a, b, c}$, Lingyu Sun $^{a}$, Feika Bian ${ }^{a}$, Yu Wang ${ }^{a}$, Yuanjin Zhao ${ }^{a, b, d *}$

${ }^{a}$ Department of Rheumatology and Immunology, Institute of Translational Medicine,

The Affiliated Drum Tower Hospital of Nanjing University Medical School, Nanjing 210008, China

${ }^{\mathrm{b}}$ State Key Laboratory of Bioelectronics, School of Biological Science and Medical

Engineering, Southeast University, Nanjing 210096, China

c Oujiang Laboratory (Zhejiang Lab for Regenerative Medicine, Vision and Brain Health), Wenzhou Institute, University of Chinese Academy of Sciences, Wenzhou, Zhejiang 325001, China

${ }^{\mathrm{d}}$ Institute for Stem Cell and Regeneration, Chinese Academy of Science, Beijing 100101, China

*E-mail: yjzhao@seu.edu.cn 

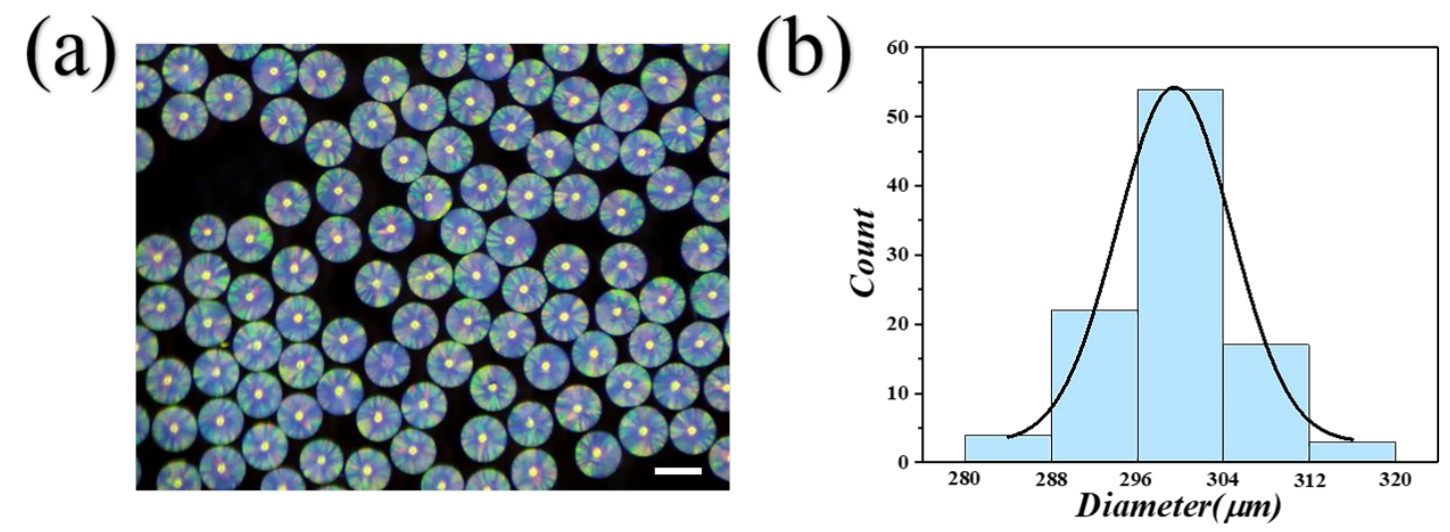

Figure S1. Analysis statistics on PhCs sizes. (a) PhCs fabricated by microfluidic chips.

(b) Graph of analysis statistics on PhCs sizes. Scale bar is $300 \mu \mathrm{m}$.

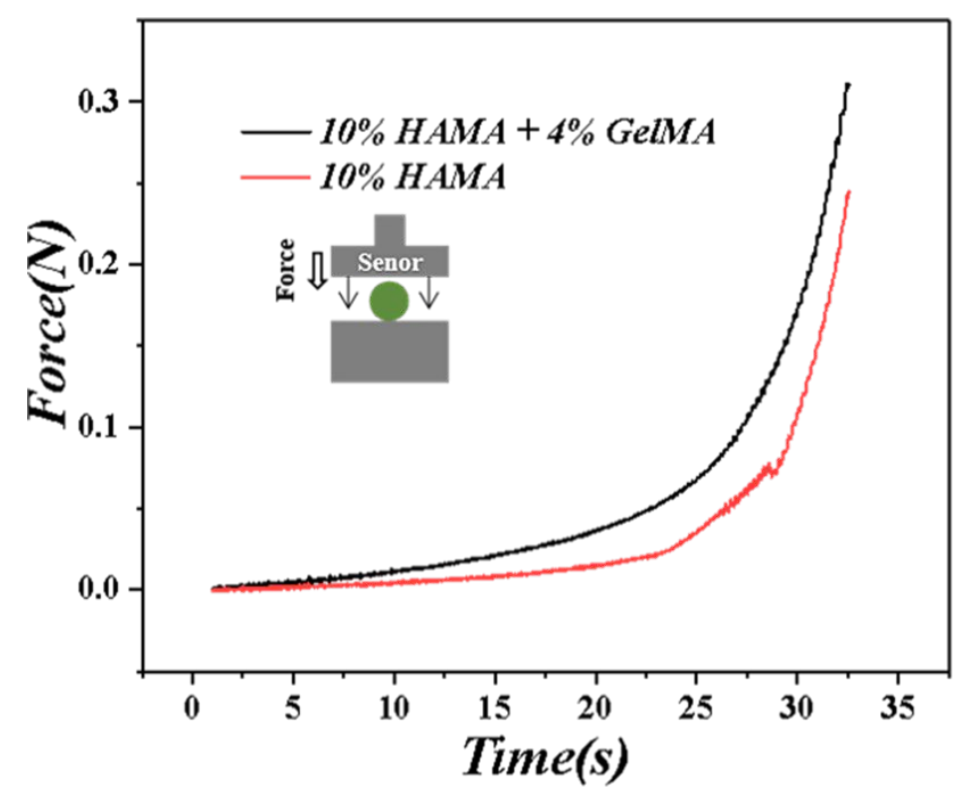

Figure S2. The axial forces of inverse opal templates. 
(a)

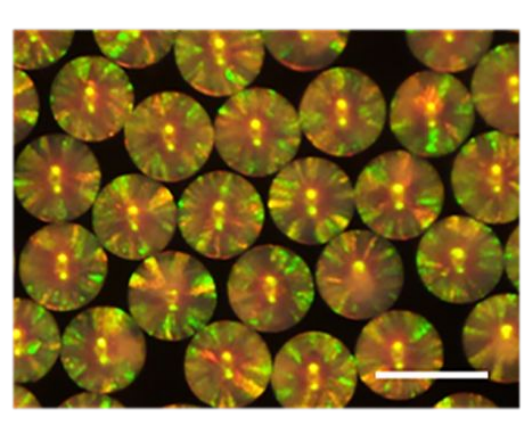

(c)

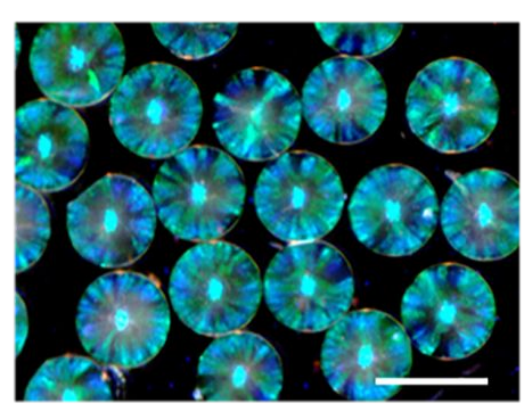

(b)

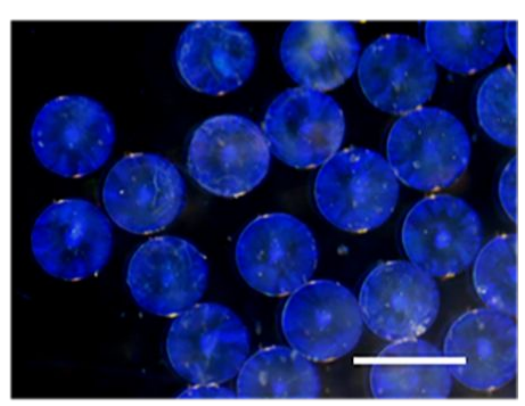

(d)

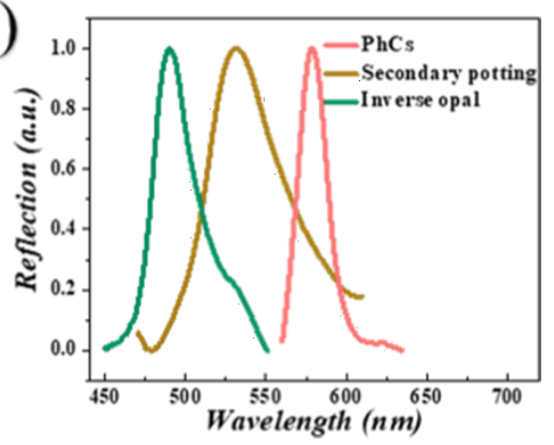

Figure S3. Images and reflection peaks of microparticles. (a-c) Images of $\mathrm{PhCs}$ templates, HAMA/GelMA inverse opal particles, GT/CG-filled hybrid particles. (d) Reflection peaks of the three different kinds of microparticles. Scale bars are $500 \mu \mathrm{m}$ in (a-c). 
(a)

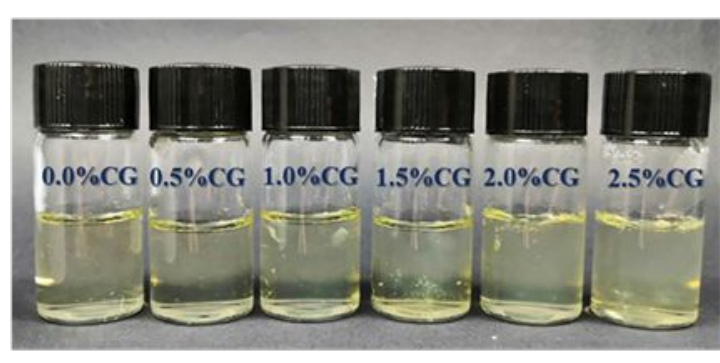

(b)

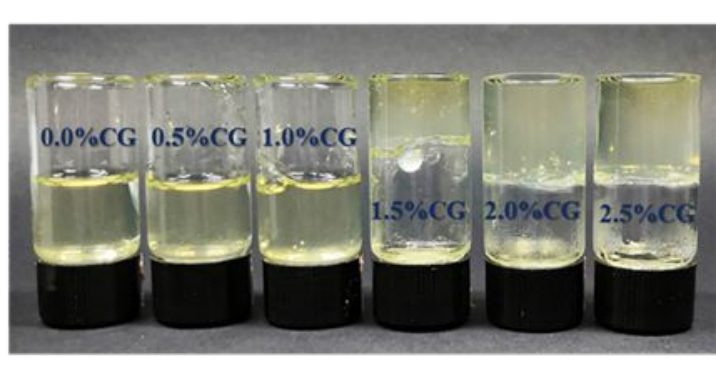

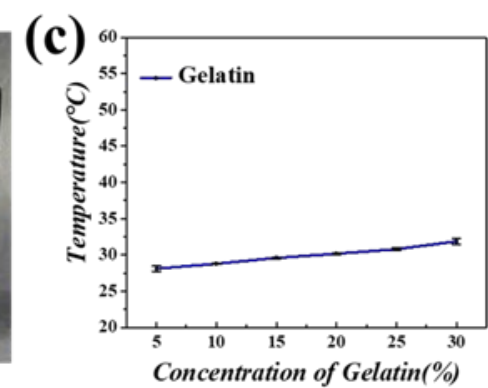

(d)

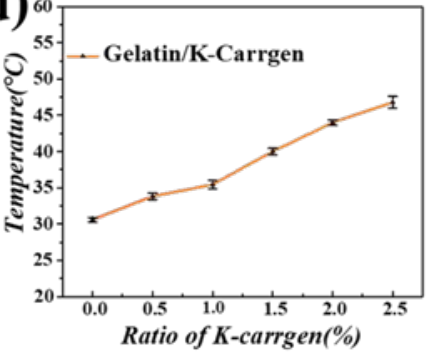

Figure S4. Images of phase-changing GT solution with different concentration of CG in the upright (a) and inverted (b) position, (c) Melting point of gelatin gel with different concentration. (d) Melting point of mixed hydrogel with different K-Carrgen ratio, the final concentration of mixed hydrogel was all $20 \%$. 

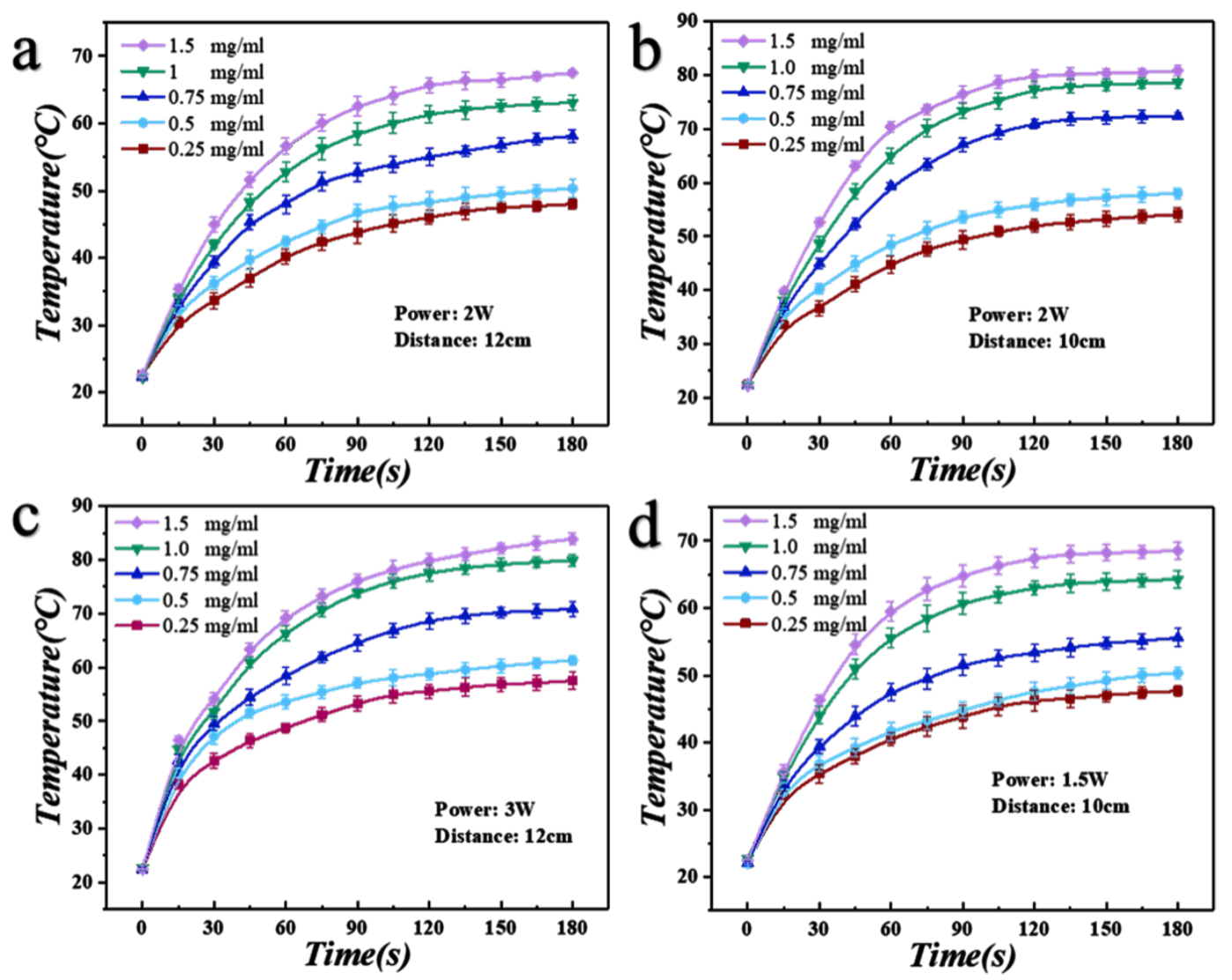

Figure S5. The relationship between the scaffold temperature and NIR irradiation time.

The NIR power was $2 \mathrm{~W}$ and distance was $12 \mathrm{~cm}$ in (a); the NIR power was $2 \mathrm{~W}$ and distance was $10 \mathrm{~cm}$ in (b); the NIR power was $3 \mathrm{~W}$ and distance was $12 \mathrm{~cm}$ in (c); and the NIR power was $1.5 \mathrm{~W}$ and distance was $10 \mathrm{~cm}$ in (d). 

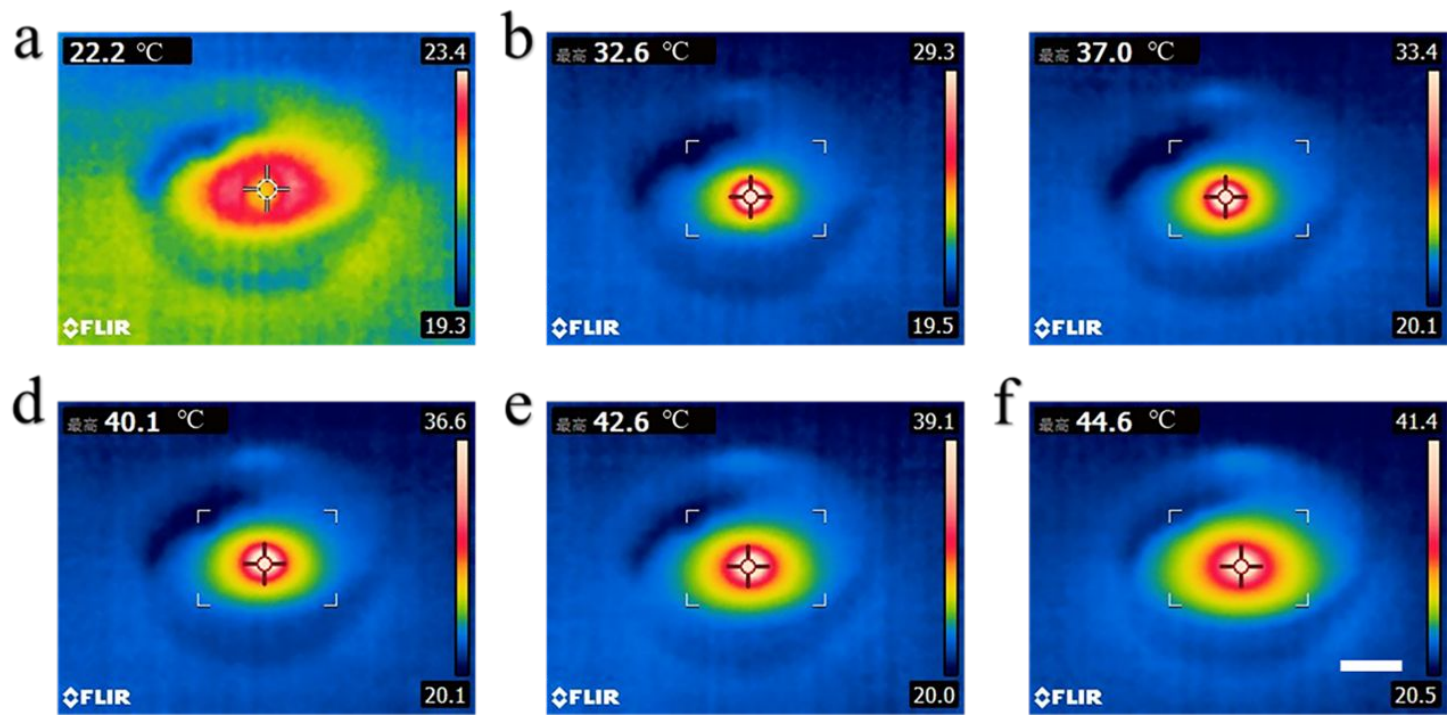

Figure S6. The real-time thermal image of the $0.5 \mathrm{mg} / \mathrm{mL}$ GO doped hydrogel beads under $2 \mathrm{~W}$ of NIR power at the distance of $10 \mathrm{~cm}$. The real-time temperature after the NIR irradiation of $0 \mathrm{~s}$ in (a), $15 \mathrm{~s}$ in (b), $30 \mathrm{~s}$ in (c), $45 \mathrm{~s}$ in (d), $60 \mathrm{~s}$ in (e) and $75 \mathrm{~s}$ in (f). Scale bar is $1 \mathrm{~cm}$. 

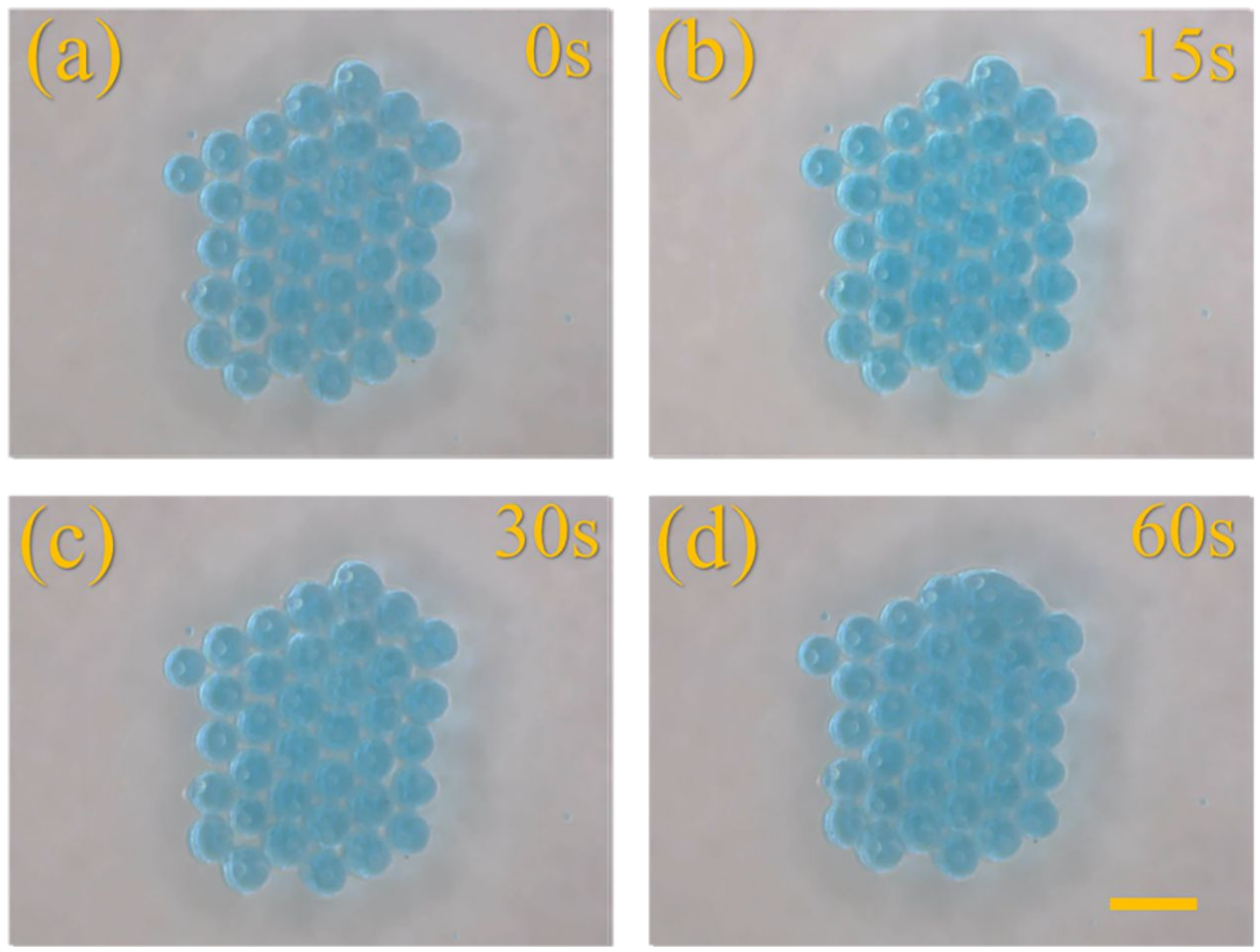

Figure S7. Images of real-time melting of pure phase-changing hydrogel particles.

Scale bar is $1 \mathrm{~mm}$. 

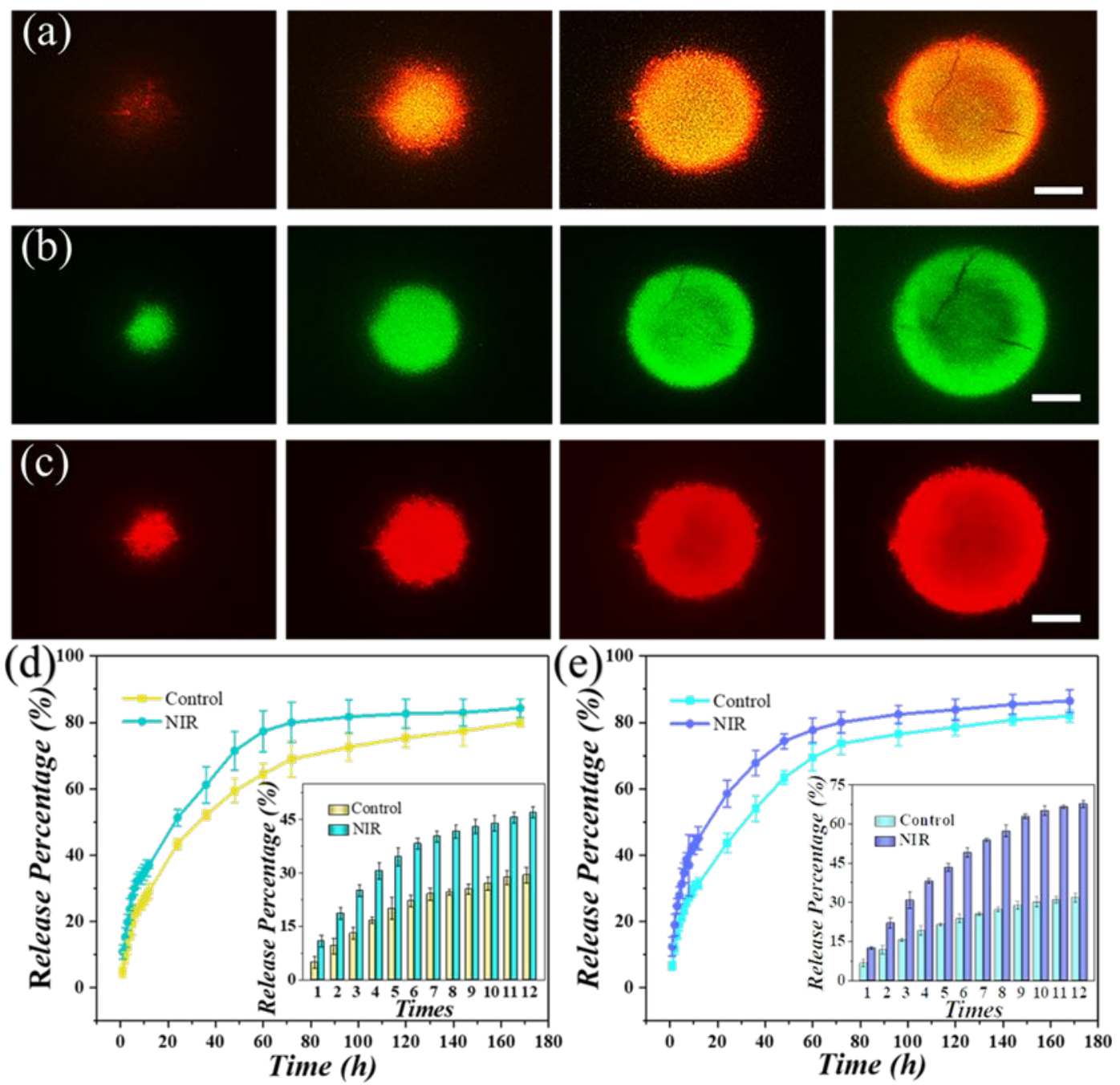

Figure S8. Drugs encapsulation and controllable release of microparticles. (a) Layerby-layer confocal laser scanning images of the FITC-BSA (EX: 490 nm; EM: $525 \mathrm{~nm}$ ) and Rhodamine B (EX: 555 nm; EM: $580 \mathrm{~nm}$ ) loaded particles under dual channel. (b, c) LSCM images of the FITC-BSA-loaded (b) and Rhodamine B-loaded (c) particles under signal channel, respectively. (d) Long term (out) and short term (in) sustained release of FITC-BSA. (e) Long term (out) and short term (in) sustained release of Rhodamine B. In the short-term release, the NIR group was irradiated once an hour, while in the long-term release, the NIR group was irradiated once a day. Scale bars are $100 \mu \mathrm{m}$ in (a-c). 


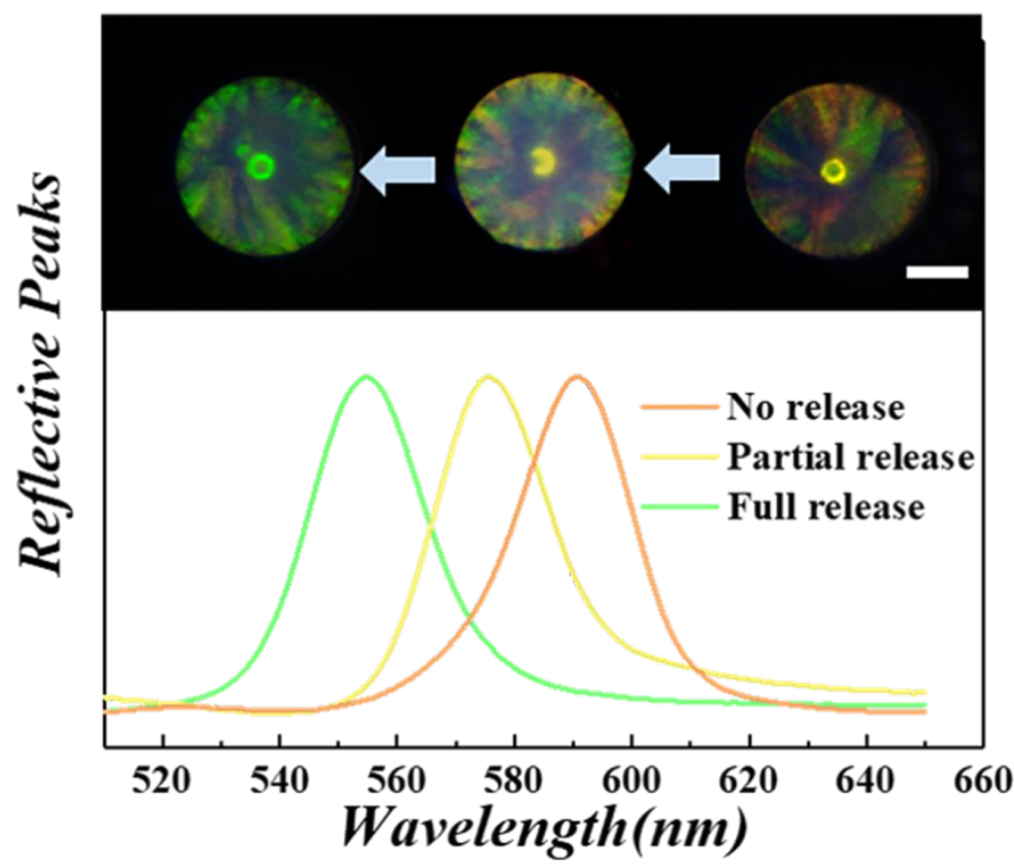

Figure S9. Structural color sensing of drugs-loaded particle in drugs release and corresponding reflection peak change. Scale bar is $100 \mu \mathrm{m}$. 


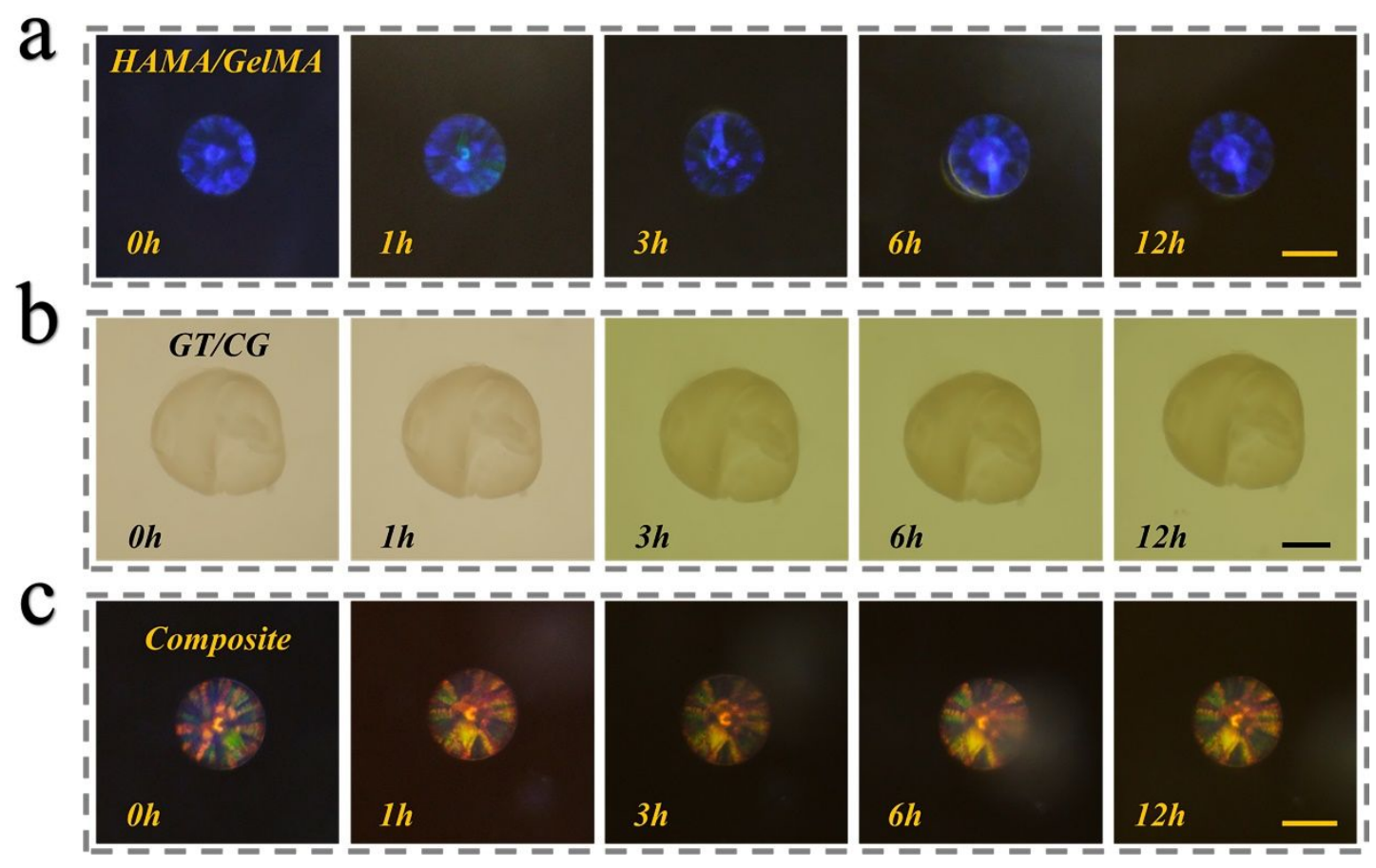

Figure S10. Swelling of hydrogel particles in PBS buffer at room temperature. (a) HAMA/GelMA inverse opal; (b) GT/CG phase-changing hydrogel particles; (c) Regluing particles. Scale bars are $200 \mu \mathrm{m}$. 
(a)

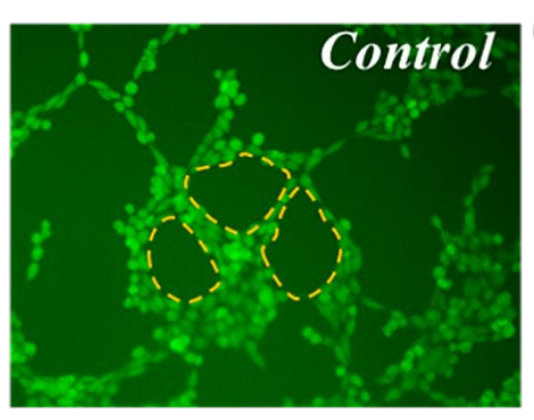

(c)

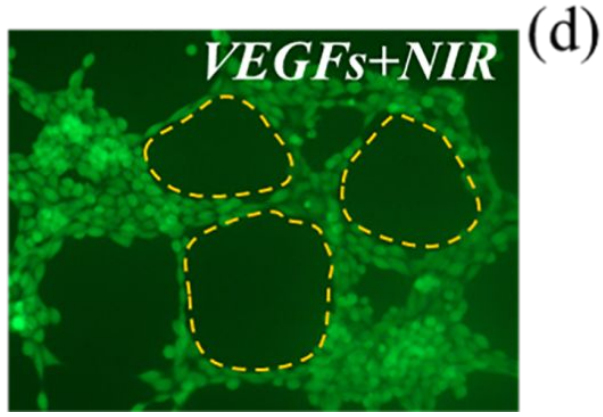

(b)
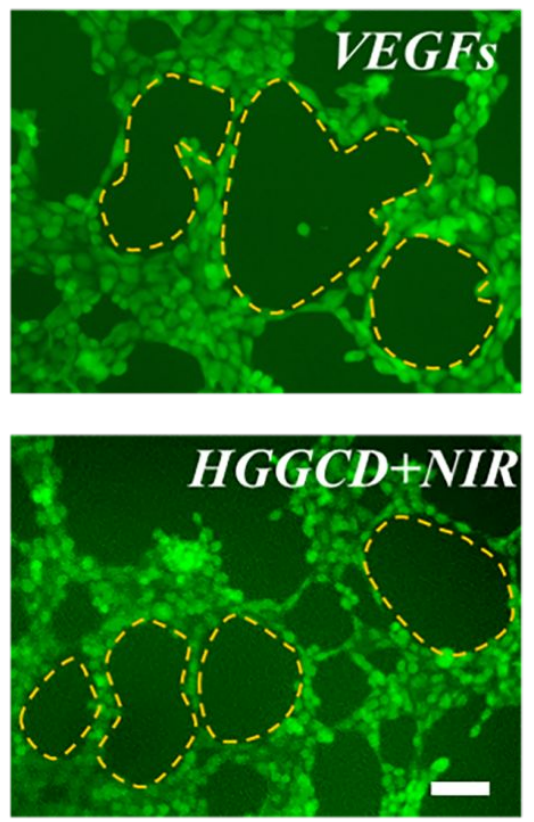

Figure S11. Fluorescent images of tube formation in different groups. (a) Control group.

(b) VEGFs group. (c) VEGFs+NIR group. (d) HGGCD+NIR group. Scale bar is $50 \mu \mathrm{m}$. 
(a)
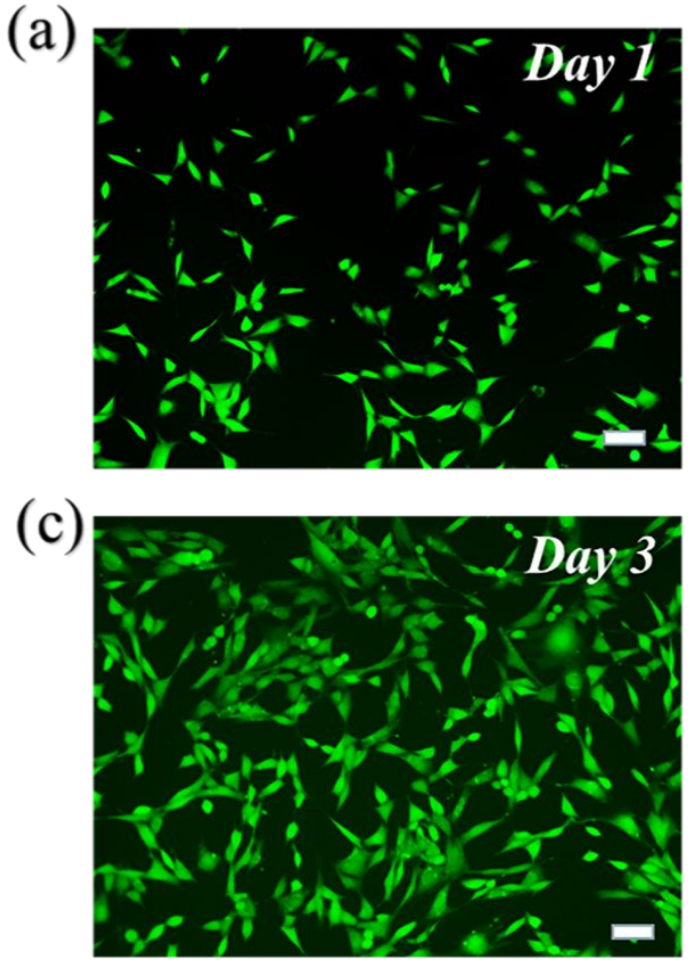

(b)

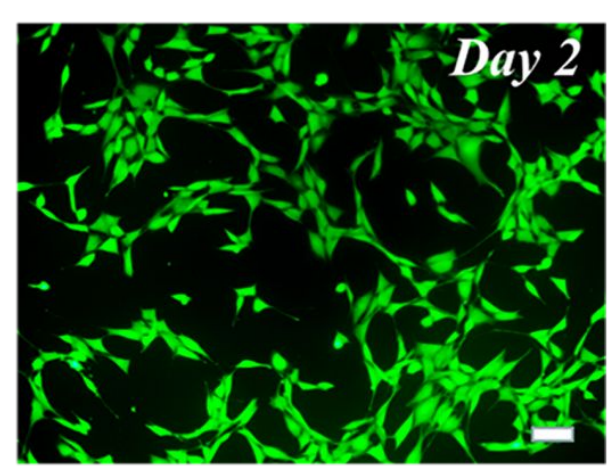

(d)

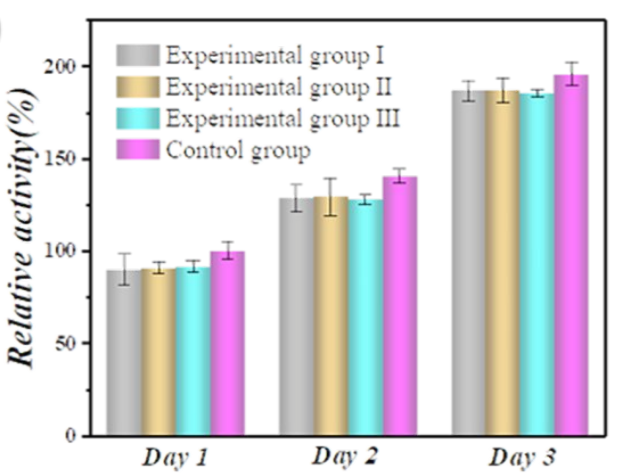

Figure S12. Biocompatibility study of materials. (a-c) The growth of 3 T3 cells cultured in the experiment group III on different days. The media in each experimental group was different, including GelMA/HAMA inverse opal scaffold (group I ), GT/CG (group II ) and mixed materials (group III). Scale bars are $100 \mu \mathrm{m}$. (d) Relative activity of $3 \mathrm{~T} 3$ cells in different groups on each day. The media in each experimental group was different, including inverse opal scaffold (group I ), GT/CG (group II) and mixed materials (group III). 

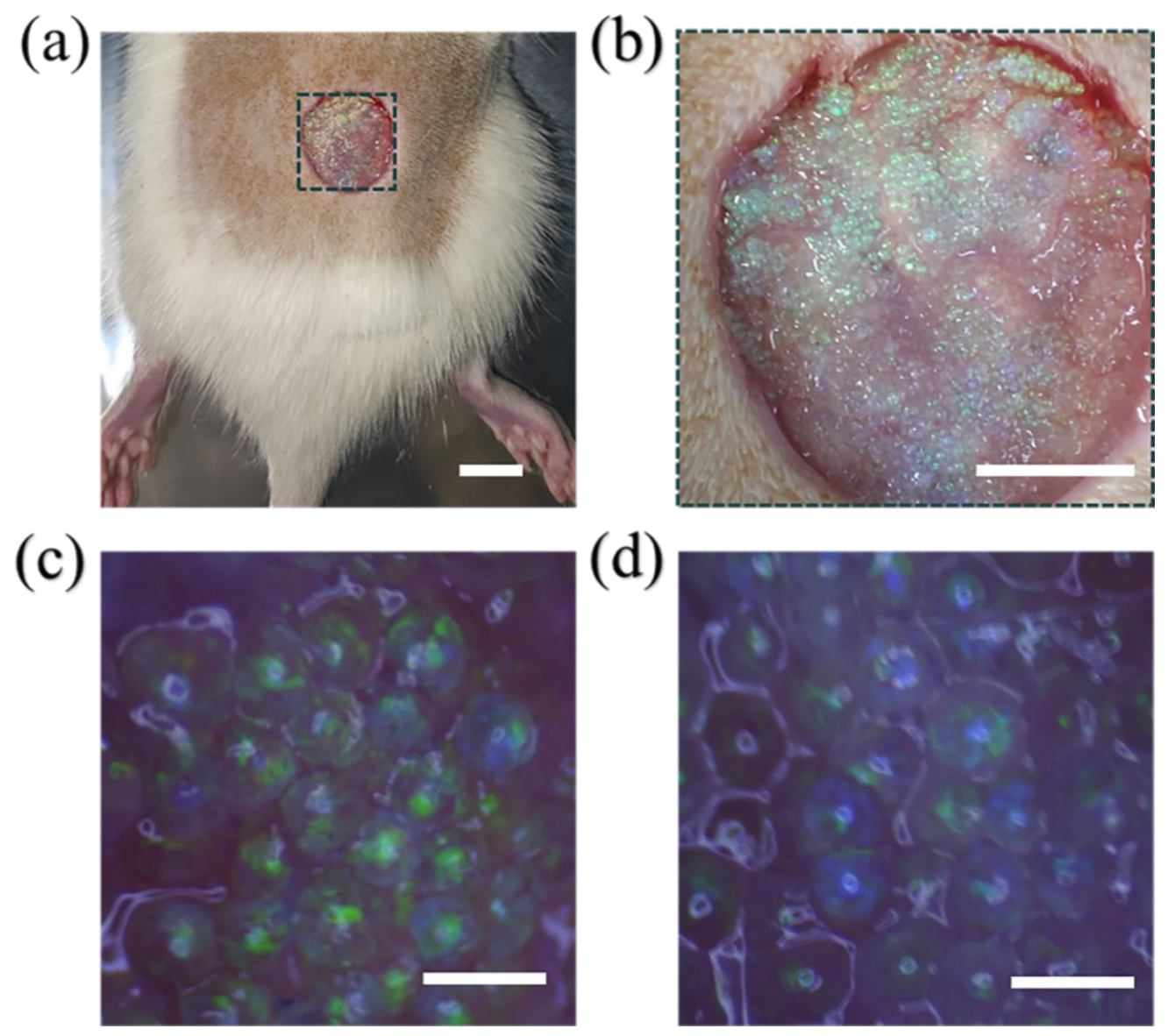

Figure S13. Composite hydrogel particles served as flexible patch on the wound. (a)

Image of treated rat with patch covering. (b) Local magnification of the wound surface from (a). (c-d) Structural color change of microparticles applied on the wound. Scale bars are $1 \mathrm{~cm}$ in (a), $5 \mathrm{~mm}$ in (b) and $500 \mu \mathrm{m}$ in (c-d).

Movie S1. Real-time video of the melting of pure phase-changing hydrogel particles. 